\title{
Disability and its prevalence and cause in northwestern Ethiopia: evidence from Dabat district of Amhara National Regional State: A community based cross-sectional study
}

\section{Solomon Mekonnen Abebe ( $\nabla$ solomekonnen@yahoo.com )}

University of Gondar College of Medicine and Health Sciences

\section{Ansha Nega}

Addis Ababa University

\section{Zemichel Gizaw}

University of Gondar College of Medicine and Health Sciences

\section{Mulugeta Bayisa}

University of Gondar College of Medicine and Health Sciences

\section{Solomon Fasika}

University of Gondar College of Medicine and Health Sciences

\section{Molalign Belay}

University of Gondar College of Social Sciences and Humanities

\section{Abel Fekadu}

University of Gondar College of Medicine and Health Sciences

\section{Mikyas Abera}

University of Gondar College of Social Sciences and Humanities

Research article

Keywords: disability, PwDs, DHSS, household survey, northwestern Ethiopia

Posted Date: April 3rd, 2020

DOI: https://doi.org/10.21203/rs.3.rs-18602/v1

License: (c) (i) This work is licensed under a Creative Commons Attribution 4.0 International License. Read Full License 


\section{Abstract}

Background: Disability is not just a physical condition; it develops through human interactions and reflects the social fabric of communities. Despite the magnitude of the problem, however, both awareness and scientific information on disability and the conditions of persons with disabilities (PwDs) are lacking in Ethiopia. Against this backdrop, the University of Gondar established the Dabat Demographic Health Surveillance System to gather longitudinal and policy relevant empirical data on various aspects of healthy living, including disability, in Dabat district of northwestern Ethiopia.

Method: A community-based cross-sectional study was conducted on 13 Kebeles of Dabat district where the DHSS operates. Quantitative data was gathered using the World Health Organization's Disability Assessment Schedule (WHODAS 2.0) and International Classification of Functioning, Health and Disability (ICF). The data was organized, analyzed, presented and described using frequencies and percentages in table and figures. Relevant variables were used to construct a logistic regression model that predicts the likelihood of disability whereby P-value $<0.05$ was considered as statistically significant.

Results: The household survey covered 17,000 households with 71,916 members amongst whom 36,462 $(50.7 \%)$ were females and $20,264(28.18 \%)$ had attended formal education. The overall prevalence of disability was $2.14 \%$ [ $95 \% \mathrm{Cl}: 2.03,2.24]$. About $8.3 \%$ of households reported at least one case of disability. Of 1537 respondents with disability, $8.19 \%$ had multiple disabilities. The visually impaired represented the highest proportion of PwDs (51\%). Generally, the major causes of disability (83\%) were modifiable; and illness (36.93\%), injury (17.81\%), and congenital (10.86\%). Advanced age [AOR=1.0; $95 \%$ Cl: $1.03,1.04]$, the illiterate [AOR $=1.15 ; 95 \% \mathrm{Cl}: 1.57,2.13]$, the unmarried/single [AOR: $1.39 ; 95 \% \mathrm{Cl}$ : 185,2.47] and the separated [AOR: $2.78 ; 95 \% \mathrm{Cl}$ : 4.14,6.19] were more likely to have disability in the study population.

Conclusion: This article reports an increased likelihood in being impaired/disabled associated with aging; and in the study population, there was high proportion of households with PwDs. Most disabilities were visual but there were also significant numbers of mobility and hearing impairments. Most school-age PwDs did not complete secondary education, while the rate of employment was significantly low. The study also identified most causes of disability were reversable.

\section{Background}

Disability is part of a human condition that can be experienced by any person at some point in life, temporarily or permanently[1]. It is an umbrella term covering various types of physical impairments, activity limitations, and participation restrictions. A person can be considered to have a disability if and when his/her performance during activities is limited in nature, function, or quality[2]. This might include physical or intellectual disability or limitation in performing activities or roles expected of individuals within their usual environment[3]. Hence, the issue of disability consistently relates to human dignity and inclusion in society. 
The World Health Organization (WHO) and the World Bank (WB) estimated that around 1 billion (15\%) of the global population currently live with some form of disability, and about $80 \%$ of them live in developing countries where rehabilitation services are poor or non-existent[1]. These numbers are increasing due to population growth, man-made and natural disasters, war, accidents and ageing.

Information pertaining to the proportion of persons with disabilities (PwDs) in Ethiopia is highly fragmented or sometimes misleading and contradictory. For example, based on the 2007 Population and Housing Census, Ethiopian Central Statistical Authority (2007) reported that there were less than 1 million Ethiopians with some form of disability i.e. 1.2\% [4]. Conversely, WHO's World Disability Report (2011) indicates that the national prevalence of disability in Ethiopia is around 17\% [1].

Despite inconsistencies and contradictions in the literature and reports on disability information and statistics in Ethiopia, mobility, visual and hearing impairment have been reported as the top-three most prevalent forms of disabilities, in that order [5]. This prevalence has also been reported to be the same in North Gondar Administrative Zone of the Amhara National Regional State (ANRS), Ethiopia [6, 7].

Promoting inclusive and sustainable development requires removing the barriers to PwDs' inclusion and participation in their community' lives. PwDs should be able to enjoy equal opportunities to schooling and employment as well as access to health and other services. In recent years, national and global actors have pushed the issue of disability to the center and front of policies, programing and service provision to ensure that PwDs enjoy the same rights and access to opportunities as other citizens [8-10].

The causes and impacts of disability are numerous and complex. However, it is evident that the major causes of disability in Africa are communicable diseases, war, accidents, and inadequate prenatal and neonatal health care services. Studies on the continent have now established that many cases of disabilities are strongly linked to poverty, poor nutrition and restricted access to basic services such as health and schooling [7]. These factors are also relevant to the Ethiopian context where the list of causes/factors include low standard of living, malnutrition, natural and man-made disasters, accidents, infectious and non-infectious diseases, intermittent wars and violence [6, 11].

Negative attitude towards and stereotyping of PwDs have dealt huge blows to their self-esteem and social participation, which increase their dependency and societal exclusion [12]. But PwDs do not make up a homogenous group. PwDs constitutes women, children, illiterates, unemployed, etc. and their experiences could be decidedly unique. This implies that community attitude towards disability significantly relies on and varies by disability type, age, gender, level of education, and employment status of PwDs [13]. In Ethiopia, about $46 \%$ of PwDs are women [14] and they, for instance, carry the double burden of stereotypes, discrimination and economic challenges associated with being women and person with disability. Age is also a factor whereby children with disabilities (CwDs) are less likely to attend schooling or access healthcare services leaving them vulnerable to poverty and poor health that substantially reduce their quality of life $[15,16]$. As a group, without underplaying variations within, PwDs are still among the most disadvantaged segment of the society. 
With new and emerging man-made and natural disability-risk factors associated with growing interconnections and communications that connect the local with the global, proactive measures such as disability surveillance is necessary to track and examine trends over time about the conditions of PwDs to inform relevant policy decisions and delivery of social- and healthcare- services. Disability surveillance is a useful mechanism to collect and provide empirical and reliable data to identify needs, set priorities, allocate resources and design effective interventions to meet the challenges of PwDs. By doing so, it will contribute to bridging the existing knowledge gap on disability - its magnitude, types, effects, trends, etc. - in Ethiopia.

Researches have documented the complicated relationship between disability and its social and health outcomes, and how the provision of long-term everyday care for PwDs is becoming a major public health problem especially for developing countries where resources, qualified personnel and rehabilitation centers and services are very limited. It is against this backdrop that University of Gondar (UoG) launched the Dabat Health Surveillance System (DHSS) in 1995 to collect, organized and disseminate regular and up-to-date information on the health conditions of the population (including PwDs) in Dabat district. This article is based on the analysis and interpretation of data gathered from households covered in DHSS longitudinal study to assess the prevalence, types, distribution, and associated factors of disability in Dabat district.

\section{Methods}

\section{Study Area}

This paper discusses disability and conditions of PwDs in Dabat district, northwestern Ethiopia, using DHSS longitudinal study as a framework. Dabat town is located $60 \mathrm{kms}$ northwest of Gondar city, and it serves as an administrative center of the Dabat district. The district has an estimated population of 168,331 (male/female: $50 \%$ ) (CSA 2013) that occupy $1,199.15 \mathrm{~km}^{2}$. It is administratively organized under 5 urban and 27 rural Kebeles with altitudes ranging between 1000 and 3000 meters above sea level (Dabat Rural Project Statistics, 2015).

The district has 29 health posts, 3 health stations and 2 health centers. UoG established DHSS in 1995 to collect demographic, social and health data in 13 Kebeles (9 rural and 4 urban) of 32 Kebeles of the district. According to the 2014 Re-senses Baseline Survey, DHSS collects data on 17,000 households, with 72,000 inhabitants, semi-annually.

\section{Study Design}

To identify the prevalence, causes and associated factors of disability at Dabat district, the study used a community-based cross-sectional design from January to June 2016.

Study Population 
All members and permanent residents of the 17,000 households under the DHSS longitudinal study make up the study population. Data on disability was gathered from household heads residing in the 13 Kebeles that were under the DHSS catchment area. The DHSS involves a set of operations to collect data on well-defined entities or primary subjects (individuals, households, and residential units) and disability on a regular-interval and over time. Semi-annually, DHSS staff and enumerators visit every household and its members in the 13 Kebeles to record their relevant health and demographic data and changes between recordings.

This article reports the findings of a separate cross-sectional study on the same study population as for the Dabat DHSS longitudinal survey, but employing robust tools to collect detailed household data and identify the prevalence, causes and associated factors of disability vis-à-vis socio-demographic and other relevant dimensions.

Sampling: inclusion and exclusion criteria

The study included all households and their members - including those on streets - in the 13 Kebeles within the Dabat DHSS catchment area. It excluded those who were not permanent residents (less than 6 months, as per the CSA definition of 'household members') during the field research period.

Data collection: tools and procedure

The household survey employed a structured and pre-tested questionnaire. The household survey questionnaire included items from The World Health Organization Disability Assessment Schedule (WHODAS 2.0), International Classification of Functioning, Health and Disability (ICF) and Medical Outcomes Study-Short Form (SF-36) tools, which were adapted based on local context and translated into the local language - Amharic.

WHODAS 2.0 is standardized and validated tool to assess health and disability status both at individual and population levels, and across cultures [17]. As a generic tool, it is effective to assess activity limitations and participation-restriction due to disability in daily lives of individuals both at population and clinical set-up. It has also been used to measure the effectiveness of disability interventions [17]. The household survey included items from WHODAS 2.0 to assess mainly the six domains of disability: cognition, mobility, self-care, getting along, life activities and participation. The tool was administered through face-to-face interview. Furthermore, items from International Classification of Functioning, Health and Disability, Children \& Youth version (ICF-CY) ware used to assess disability among population under 18 years of age. Survey data were collected by well-trained data collectors and DHSS staffs. They visited all 17,000 households in Dabat district to collect data on every member of the household using the questionnaire. Heads of households were interviewed to collect the necessary information on all members of the household, including themselves. When a member of a household was reported to manifest signs of disability, data collectors administered a checklist (WHODAS 2.0 or ICF-CY and SF-36) to assess its nature, magnitude and quality of life. Finally, when new cases of disability were identified or reported, they were referred and/or linked to local Community-based Rehabilitation (CBR) fieldworker. 
Data processing and analysis

Survey data was entered into and analyzed using STATA (v.12) software. Descriptive statistics - means, percentages, and standard deviation, etc. - were employed to describe the characteristics of the study population. Table and figures were used to present aggregated and disaggregated, as required, data. Logistic regression models were constructed to predict factors associated with disability among the study population. A P value of $<0.05$ was considered statistically significant.

\section{Results}

As Table 1 shows, survey data was collected on 71,916 members of 17,000 households. $36,462(50.7 \%)$ were females, and 54,489 (75.77\%) lived in rural areas. The survey also identified that $22,274(30.97 \%)$ cannot read and write, while 22,133 (30.78\%) and 26,394 (36.7\%) were aged, respectively, under 14 years and 18 years.

The overall prevalence of disability in the 13 Kebeles was 2.14\% [95\% Cl: 2.03, 2.24]. Higher prevalence was noted among females (2.29\%) than males (1.97\%). As Table 2 and Fig. 1 reveal, the prevalence of disability tends to increase with increase in age, and it is the highest among persons of 65 and above years old $(10.18 \%)$. A reverse trend was observed for the prevalence of disability vis-à-vis educational status i.e. the highest proportion of disability - $971(63.2 \% \%)$ - was recorded for those who cannot read and write, and the difference is stark when we compare it to those with Grade 11/12 (0.72\%) and Grade $12^{+}(0.98 \%)$ education (Table 3$)$.

The proportion of reported PwDs per household was 8.3\% (1411 per 17,000). The magnitude of reported disability was high among those with 'separated' marital status (63 (10.21\%)), followed by the widowed (176 (9.33\%)). By occupation, the highest level of disability was observed among the unemployed (5.42\%). There was also variation between urban (2.08\%) and rural (2.15\%) Kebeles of Dabat district in terms of the percentage of PwDs in the respective populations (Table 1).

$8.19 \%$ of the 1,537 PwDs identified in the study reported more than one type of disability. In terms of single-disabilities, $51 \%$ had visual, while $24.3 \%$ and $22.3 \%$ had mobility and hearing disabilities. Noticeably, there was a marked increase in hearing and visual disabilities among the ageing household members (Table 2 \& Fig. 2).

Only 253 (16.5\%) PwDs attended any form of formal education. In fact, as educational level increases, the proportion of PwDs attending higher grades fell significantly, and, consequently, less than $1 \%$ of PwDs had completed secondary education (Table 3).

With regard to occupation, there was small proportion of government employees both in urban $(2.03 \%)$ and rural $(0.35 \%)$ areas. More men than women worked on farms, and farming was reported as the main occupation by both urban (17\%) and rural (49.8\%) areas (Table 4$)$. 
The most frequently reported cause of disability was illness (36.93\%), followed by accidents $(17.81 \%)$, unknown (11.57\%), congenital (10.86\%) and ageing (5.2\%). However, we should note here that more than $83 \%$ causes of disabilities were modifiable (preventable or treatable) factors. (Table 5 and Fig. 4)

Multivariate logistic regression analysis showed that ageing [AOR $=1.0 ; 95 \% \mathrm{Cl}: 1.03,1.04]$, inability to read and write $[\mathrm{AOR}=1.15 ; 95 \% \mathrm{Cl}: 1.57,2.13]$, and marital status i.e. being single [AOR: $1.39 ; 95 \% \mathrm{Cl}$ : 185,2.47] and separated [AOR: $2.78 ; 95 \% \mathrm{Cl}: 4.14,6.19]$ were significant predictors of disability in the study population (Table 6).

\section{Discussion}

This study found that though the general prevalence of disability was $2.14 \%$, its proportion increased significantly among older people. The proportion of households with members with disability i.e. $8.3 \%$ was high when compared to previous studies on the study area. As questionable they were in reliability, representativeness or timeliness, CSA [18] and Chala [19] reported $1.82 \%$ and $1.09 \%$, respectively, disability prevalence for Dabat district, which were significantly lower than the $2.14 \%$ reported in this study. Conversely, however, $2.14 \%$ is significantly lower than an estimated $17.6 \%$ national disability prevalence rate in Ethiopia that WHO and WB $(2011)$ reported $[1,9]$.

There are several possible explanations why different sources provide different reports on disability prevalence. For one, WB and WHO operate with a broad definition of disability and include various factors, such as chronic illnesses, within the rubric of 'disability.' In the context of developing countries with limited resources for rehabilitation services and supporting PwDs, pragmatic and contextual reasons dictate operationalizing disability in narrow terms to avoid unrealistic considerations and recommendations for policy formulation and social work practice. For another, even with a narrow definition of disability in our study, the design of the questionnaire, lack of knowledge in nature and degree of one's disability, and negative attitude towards disability and PwDs may have lowered reports on disability in the study area.

In terms of type of disability, the current study reported similar result as the previous study in Dabat district undertaken by the same team of researchers [19]. Both studies found visual impairment affects the highest proportion of PwDs; but while the current study found that mobility and hearing impairments are second and third in the number of people they affect, their relative proportion was reversed three years earlier[19].

A small fraction of PwDs attended formal education; and more importantly, with advance in educational level, their progression to higher grades decreases significantly. This result was consistent with the Handicap International report that indicated a small fraction (3\%) of the estimated 2.4 to 4.8 million school-age CwDs went to school due to underlaying reasons that include stigma among parents and educators towards CwDs, school inaccessibly, rigid teaching practice, poorly trained teachers to accommodate the special needs of CWDs and lack of adaptive hearing resources [20]. Furthermore, a study on PwDs in different ages and their families in South Africa documented how they live with stigma 
and social exclusion and more than $80 \%$ of them reside in rural areas [21]. Other studies [15, 22] also report CWDs are less likely to attend schooling and access health care, and they are more vulnerable to poverty which may result in substantial decline in their quality of life.

This study reported that the majority disabilities were due to modifiable causes; and the causes and impacts of disability are multifaceted. Concurrent to these findings, studies have documented that the majority of disabilities in Africa result from illness, injury, and accidents - causes that are preventable [7, 23]. Most disability can be linked to poverty, and restricted access to basic services [24-26]. Besides, inadequate health care services were also significant contributors to the disability problem on the continent [17]. Similarly, this study reported a very small proportion of PwDs employed in the government

- both in urban and rural areas - and a significant majority of them were involved in small-scale farming with strained livelihoods.

The relationship between health outcomes and disability is complicated and the provision of long-term care in everyday life for people living in resource limited settings is a major public health problem especially in developing countries where the prevention and the health service is very scarce[16]. Moreover, negative attitude towards and stereotyping of PwDs limit educational and employment opportunity that creates dependency and exclusion of PwDs [27], and this was noted in the current study.

When it comes to women with disabilities, they carry the double burden of stigma and discrimination and economic problem due to their gender-roles and disability [14]. Disability and poverty exacerbate their socioeconomic wellbeing and their general quality of life.

Strengths and Limitations

The study aimed at exploring factors and the prevalence of disability and its deleterious impacts in low resource setting i.e. Dabat district, Northwestern Ethiopia. Large size samples were drawn to collect representative empirical data about the population. Literature review revealed that there is dearth of scientific, empirical information to understand the trend and changes on the prevalence and magnitude of disability and its effects in Ethiopia. It has also established the evident fact that there is low community and governmental attention on disability and the empowerment of PwDs in developing countries such as Ethiopia. Therefore, by identifying contributing factors to disability and its prevalence and impacts on peoples' lives in northwest Ethiopia, this study will promote disability awareness and inclusive society as well as inform the agenda for disability research, policy and practice in the district and beyond. Conclusion

This study found the proportion of reported disability per household to be high. Vision and mobility disability were the most frequently observed types of disability, and most causes of disability were modifiable. This study indicated that the prevalence of disability increase among the ageing-groups. As educational level increase, the proportion of PwDs attending school decreases, and most PwDs do not complete secondary education. Similarly, a small proportion of PwDs were government employees, and the majority were engaged in farming. 
Early intervention and appropriate medication will prevent the burden of disability. To this end, appropriate attention should be given to improve quality of healthcare facilities and services to reduce the burden of disability on families, communities and the health care system. Moreover, community awareness creation and enhancing inclusive education will improve education opportunity for PwDs. With improve in educational level and skills, PwDs will be able to find and/or create jobs thereby promoting their independence and better quality of life. Further qualitative research is recommended to examine contextual factors that influence the social participation and quality of life of PwDs in the context of Dabat district.

\section{Abbreviations}

ANRS

Amhara National Regional State

CBR

Community-based Rehabilitation

CSA

Central Statistical Agency

CwDs

Children with Disabilities

DHSS

Demographic Health Surveillance System

ICF

International Classification of Functioning, Health and Disability

ICF-CY

Health and Disability, Children \& Youth version

NGZ

North Gondar Zone

PwDs

Persons with Disabilities

WB

World Bank

WHO

World Health Organization

WHODAS 2.0

World Health Organization Disability Assessment Schedule

\section{Declarations}

\section{Ethical considerations}


The University of Gondar's Institutional Review Board (UoG-IRB) cleared the research for meeting required and appropriate ethical standards. Two support letters - from the Vice President's Office for Research and Community Services and the College of Medicine and Health Sciences - were submitted to Dabat District Administrator. Letter of information describing the purpose and objectives of the study, and written informed consent was obtained from the study participant (household head), and consent forms were given or read to study participants prior to the commencement of the interview. Participants were granted the right to terminate their participation in the study at any point if and when they chose to.

Once in the field, researchers and DHSS staff put their maximum efforts in assuring all ethical and scientific standards are maintained. Participants' privacy and confidentiality were respected during and after interviews. Once data was collected, each questionnaire was coded, and personal identifiers were removed to maintain anonymity and confidentiality of participants. All data was entered and saved on a computer with a strong password which was only accessible by the research team. Participants who reported disability or were found ill during data collection were either linked to UoG-CBR fieldworkers or health centers for better care.

Consent for publication: "Not applicable"

Data sharing statement: Since the data presented in this report are the part of the large DHSS survey data, we have to abide by the data sharing policy of University of Gondar; nonetheless, we have included all important information regarding data presented on the tables and figures (No additional data are available).

Competing interests: The authors declare no financial and non-financial competing interests

Funding: University of Gondar.

\section{Authors' contributions}

- SMA, MB, MB, SF, AN, MA, Z, WW, AF, designed the study;

- SMA, performed the analysis and interpretation of data; and,

- SMA, MB, MB, SF, AN, MA, Z, WW, AF, drafted and finalized the write-up of the paper.

- All authors prepared the draft manuscript, read and approved the final manuscript.

\section{Acknowledgement}

We would like to acknowledge the University of Gondar for the financial support. We extend our thanks to the Dabat District Health Office for their permission to conduct the study. We want to express our gratitude to all staff of the Dabat DHS and the study participants. 


\section{References}

1. . In: World Report on Disability 2011. edn. Geneva; 2011.

2. Cohen ME, Marino RJ: The tools of disability outcomes research functional status measures. Archives of physical medicine and rehabilitation 2000, 81(12 Suppl 2):S21-29.

3. . In: Survey Measurement of Work Disability: Summary of a Workshop. edn. Edited by Mathiowetz N, Wunderlich GS. Washington (DC); 2000.

4. 2007 POPULATION and HOUSING CENSUS OF ETHIOPIA. In. Addis Ababa: Central Statistical Authority; 2012.

5. Hong T, Mitchell P, Burlutsky G, Liew G, Wang JJ: Visual Impairment, Hearing Loss and Cognitive Function in an Older Population: Longitudinal Findings from the Blue Mountains Eye Study. PloS one 2016, 11(1):e0147646.

6. Fitaw Y, Boersma JM: Prevalence and impact of disability in north-western Ethiopia. Disability and rehabilitation 2006, 28(15):949-953.

7. Tamrat G, Kebede Y, Alemu S, Moore J: The prevalence and characteristics of physical and sensory disabilities in Northern Ethiopia. Disability and rehabilitation 2001, 23(17):799-804.

8. Krahn GL: WHO World Report on Disability: a review. Disability and health journal 2011, 4(3):141-142.

9. World report on disability. Lancet 2011, 377(9782):1977.

10. Organization IL: Inclusion of People with Disabilities in Ethiopia. In.; 2013.

11. Alemu T, Fantahun M: Sexual and reproductive health status and related problems of young people with disabilities in selected associations of people with disability, Addis Ababa, Ethiopia. Ethiopian medical journal 2011, 49(2):97-108.

12. RIESER R: Issues of gender, 'race', sexuality, disability and social class: Disability equality: Confronting the oppression of the past. Taylor \& Francis press 2006.

13. Getachew AT: Attitudes of Ethiopian college students toward people with visible disabilities. University of lowa lowa Research Online; 2011.

14. Sumilo D, Kurinczuk JJ, Redshaw ME, Gray R: Prevalence and impact of disability in women who had recently given birth in the UK. BMC pregnancy and childbirth 2012, 12:31.

15. Kuper H, Monteath-van Dok A, Wing K, Danquah L, Evans J, Zuurmond M, Gallinetti J: The impact of disability on the lives of children; cross-sectional data including 8,900 children with disabilities and 898,834 children without disabilities across 30 countries. PloS one 2014, 9(9):e107300.

16. Dassah E, Aldersey H, McColl MA, Davison C: Factors affecting access to primary health care services for persons with disabilities in rural areas: a "best-fit" framework synthesis. Global health research and policy 2018, 3:36. 
17. Tarvonen-Schroder S, Hurme S, Laimi K: The World Health Organization Disability Assessment Schedule (WHODAS 2.0) and the WHO Minimal Generic Set of Domains of Functioning and Health versus Conventional Instruments in subacute stroke. Journal of rehabilitation medicine 2019, 51(9):675-682.

18. Ethiopia Demographic and Health Survey: Calverton, Maryland, USA: CSA and ICF International. In. Cover photograph: (c) 2003 Chandrakant Ruparelia, Courtesy of Photoshare 2011.

19. Chala MB, Mekonnen S, Andargie G, Kebede Y, Yitayal M, Alemu K, Awoke T, Wubeshet M, Azmeraw T, Birku $\mathrm{M}$ et al: Prevalence of disability and associated factors in Dabat Health and Demographic Surveillance System site, northwest Ethiopia. BMC public health 2017, 17(1):762.

20. Ribohn U: Human rights for persons with disabilities; an evaluation of the work plan. In. Edited by 30 : Commissioned by Sida, Department for International Organisations and Policy Support; 2013.

21. Lorenzo T, van Pletzen E, Booyens M: Determining the competences of community based workers for disability-inclusive development in rural areas of South Africa, Botswana and Malawi. Rural and remote health 2015, 15(2):2919.

22. Khoo TB, Kassim AB, Omar MA, Hasnan N, Amin RM, Omar Z, Yusoff AF: Prevalence and impact of physical disability on Malaysian school-aged children: a population-based survey. Disability and rehabilitation 2009, 31(21):1753-1761.

23. Lerner WM, Weil PA, Kirschner KL: The prevalence and impact of disability among healthcare executives. Healthcare executive 2006, 21(6):24-26, 28-29.

24. G. Tamrat YK, S. Alemu and J. Moore.: The Prevalence and Characteristics of Physical and Sensory Disabilities in Northern Ethiopia. Disability and rehabilitation 2001, 23(17, 20):799-804.

25. (CARDOS) CfARaDOS: Report on the general conditions of people with disabilities in Ethiopia. Addis Ababa. 2007.

26. (ACPF) ACPF: Educating Children with Disabilities in Africa: Towards a policy inclusion. In. Addis Ababa, Ethiopia 2011.

27. Rieser R: Disability equality: confronting the oppression of the past. In: Education Equality and Human Rights: issues of gender, 'race', sexuality, disability and social class. edn. Edited by Cole M: Routledge Taylor \& Francis press; 2006.

\section{Figures}




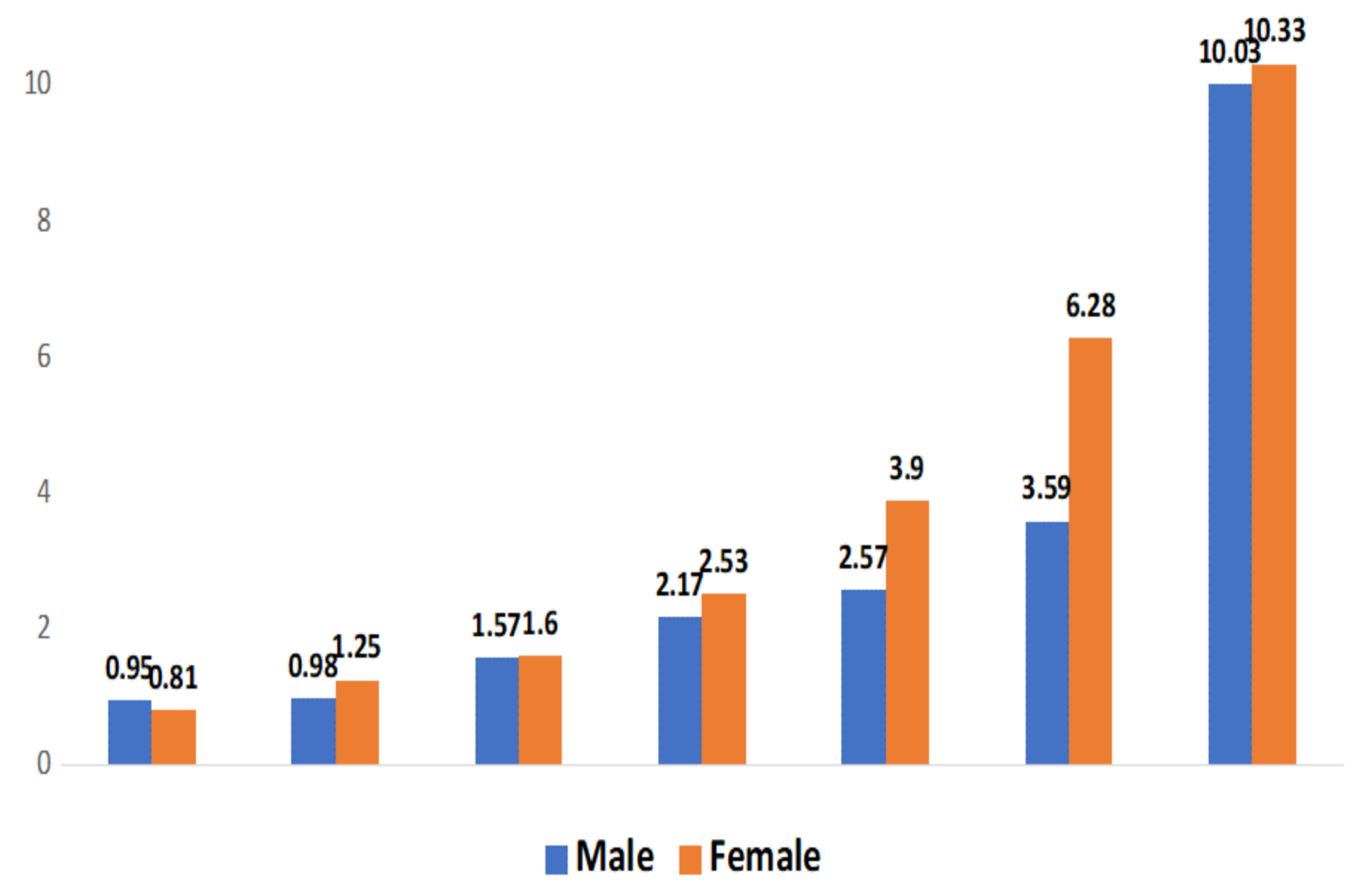

Figure 1

Magnitude of disability by sex and age in Dabat district (Source: Survey 2016) 


\section{Percentage of disability by age and type}

12

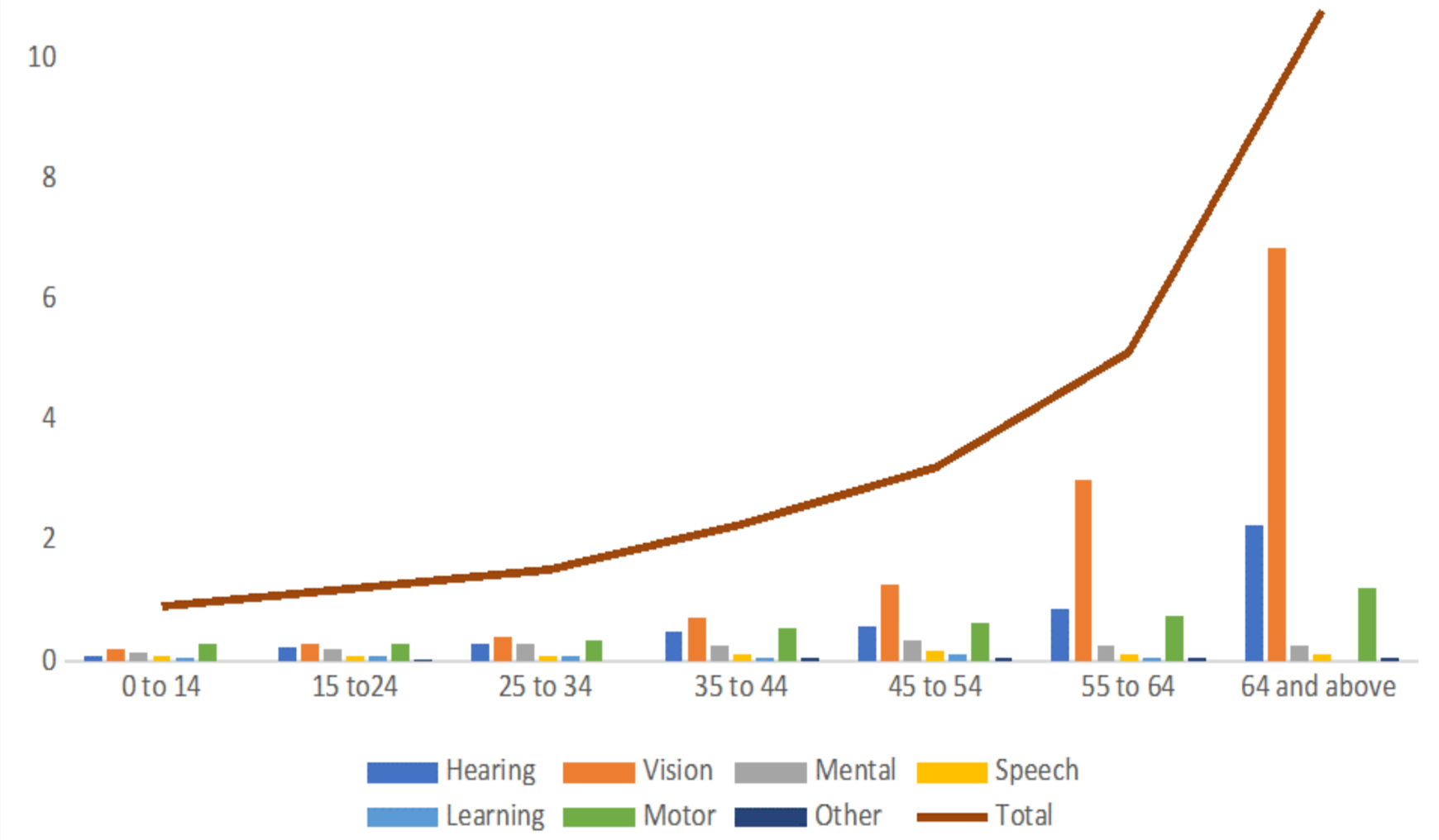

Figure 2

Distribution and type of disability by age among PwDs in Dabat district (Source: Survey 2016) 


\section{Percentage}

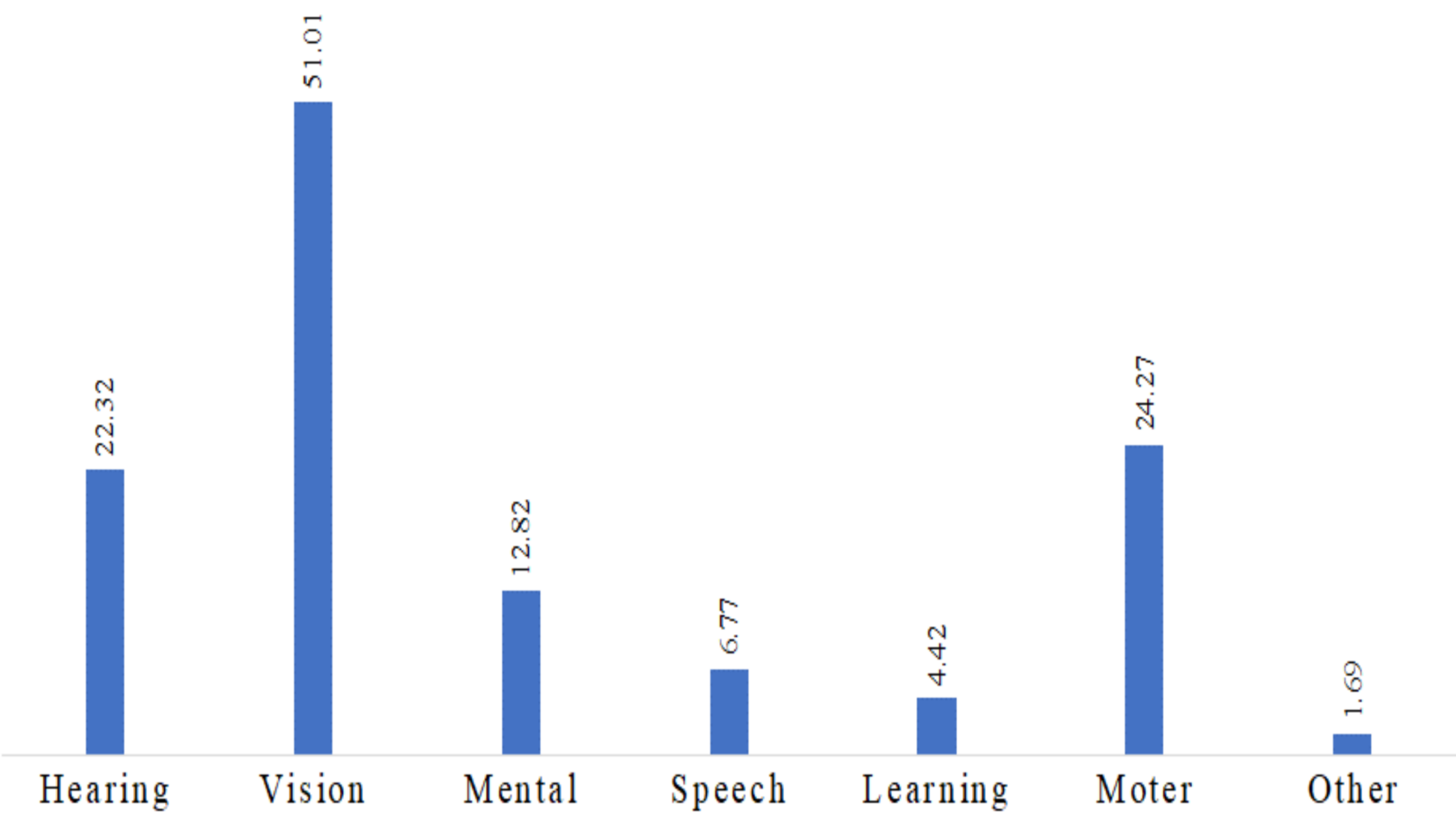

Figure 3

Types of disability among PwDs in Dabat district (Source: Survey 2016) 


\section{Persentage}

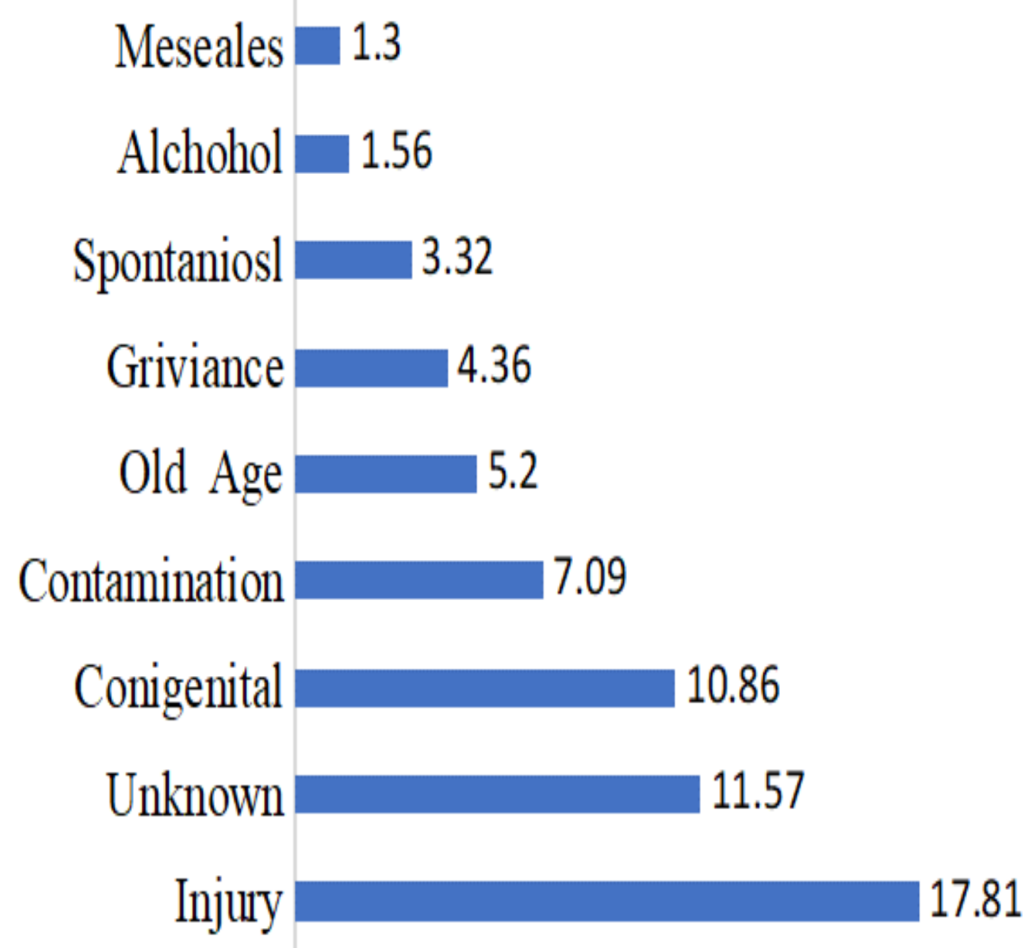

Meseales $\square 1.3$

Alchohol 1.56

Spontaniosl $\quad 3.32$

Griviance $\quad 4.36$

Old Age 5.2

Contamination $\quad 7.09$

Conigenital $\quad 10.86$

Unknown 11.57

Injury 17.81

Ilness

Figure 4

Perceived causes of disability among PwDs in Dabat district (Source: Survey, 2016). 